\title{
Cashew nut shell liquid, a valuable raw material for generating semiconductive polyaniline nanofibers
}

\author{
Raiane Valenti Gonçalves ${ }^{1}$, Mara Lise Zanini ${ }^{1}$, José Antonio Malmonge ${ }^{2}$, Leila Bonnaud ${ }^{3}$ and \\ Nara Regina de Souza Basso ${ }^{1 *}$
}

\author{
${ }^{1}$ Faculdade de Química, Pontifícia Universidade Católica do Rio Grande do Sul - PUCRS, \\ Porto Alegre, RS, Brazil \\ ${ }^{2}$ Universidade Estadual Paulista - UNESP, Faculdade de Engenharia, Ilha Solteira, SP, Brazil \\ ${ }^{3}$ Laboratory of Polymeric and Composite Materials, Materia Nova Research Center, Mons, Belgium \\ *nrbass@pucrs.br
}

\begin{abstract}
Cashew nut shell liquid (CNSL) is an abundant and renewable by-product of the cashew nut industry. It appears to be a valuable raw material for generating semiconductive polyaniline (PAni) nanomaterial with enhanced thermal stability and well-defined nanofiber morphology following a polymerization dispersion process. This study confirms that CNSL acts as a soft template during PAni synthesis, leading to an improvement in the nanofiber aspect. CNSL also improves the thermal stability of the PAni nanomaterial. Moreover, CNSL is an effective surfactant that promotes and stabilizes the dispersion of PAni nanofibers within water, allowing the more ecofriendly preparation of PAni nanomaterial by substituting the commonly used organic solvent with aqueous media. Finally, although CNSL promotes the formation of the conductive emeraldine salt form of PAni, increasing CNSL concentrations appear to plasticize the PAni polymer, leading to reduced electrical conductivity. However, this reduction is not detrimental, and PAni nanofibers remain semiconductive even under high CNSL concentrations.
\end{abstract}

Keywords: cashew nut shell liquid, nanofibers, polyaniline, semiconductive material, soft template.

\section{Introduction}

Polyaniline (PAni) in the emeraldine salt form is one of the most widely studied conducting polymers because of its easy synthesis, low cost, and acid-doping/dedoping chemistry-based property. Furthermore, the final polymer shows high stability and excellent electrical properties when exposed at environmental conditions. A limitation of PAni in the emeraldine salt form is its insolubility in common organic solvents and polymers, which limits its application as a filler in nanocomposites. Many studies have tried to overcome this difficulty ${ }^{[1,2]}$.

PAni nanostructures can be synthesized with zero dimensions (e.g., nanospheres ${ }^{[3]}$ ), one dimension (e.g., nanofibers ${ }^{[4]}$, nanorods ${ }^{[5]}$, and nanotubes ${ }^{[6]}$ ), and two dimensions (e.g., nanobelts ${ }^{[7]}$ and nanosheets $\left.{ }^{[8]}\right)$. PAni nanostructures have been widely investigated because they combine the unique characteristics of a conventional polymer with the quantum effects of nanomaterial ${ }^{[9,10]}$. Different experimental parameters such as type of dopant used, [aniline]/[soft template] ratio, $\mathrm{pH}$, temperature, and reaction time can strongly affect the PAni morphology $y^{[1,11-13]}$.

PAni nanofibers are prepared by methods such as using soft templates ${ }^{[4,14]}$ and surfactant-free emulsion polymerization $^{[15]}$. Micelle as a soft template has been regarded as an appropriate theory in order to describe the formation of the PAni nanostructure ${ }^{[16-18]}$. In this model the dopant with an amphiphilic structure form ordered templates like micelles and bi-layers to act as seeds for the growth of polyaniline chains and therefore the morphology of the final product is highly dependent on the structure of the dopant molecule and its ability to form stable micelles in water. The high surface area of PAni nanofibers allows potential applications in nanodevice manufacturing and preparation of polymeric conductive composites. Therefore, different mechanisms and methodologies for synthesizing PAni nanofibers have been studied ${ }^{[4,19,20]}$.

The use of different soft template in the PAni synthesis has been reported in literature. Yin and Yang used dodecylbenzene sulfonic acid (DBSA) as a soft template in the synthesis of polyaniline doped with hydrochloric acid $(\mathrm{HCl})$. It was observed that the morphology and electrical conductivity of the resulting PAni depend on the ratio [aniline]: [DBSA] and $\mathrm{HCl}$ concentration. PAni nanofibers and nanotubes with electrical conductivity values of $0.16 \mathrm{~S} . c m^{-1}$ and $5.3 \times 10^{-3} \mathrm{~S} \mathrm{~cm}^{-1}$, respectively, were obtained in the presence of low DBSA and $\mathrm{HCl}$ concentrations ${ }^{\left[{ }^{11]}\right.}$. Sucrose octaacetate as a soft template was also used in the synthesis of PAni doped with HCl. PAni synthesized with different sucrose octaacetate contents showed electrical conductivity values in the order of $10^{-1} \mathrm{~S}_{\mathrm{cm}} \mathrm{cm}^{-1}$, which are comparable to other conventional methods described in literature. The morphology of nanofibers or nanorods was influenced by the sucrose content and agglomerates were observed increasing the amount of the soft template ${ }^{[21]}$. In another work PAni was synthesized using $\mathrm{HCl}$ as dopant and the N-cetyl-N, N, N-trimethyl trimethylammonium bromide (CTAB) surfactant as soft template. The authors found that surfactant/oxidant ratio influenced the aniline polymerization and different morphologies and electrical 
conductivity values were obtained for PAni: nanotube, nanorod, nanosphere and nanosheet with electrical conductivity

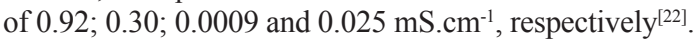

Cashew nut shell liquid (CNSL), a by-product of the cashew industry, is rich in natural nonisoprenoid phenolic lipids. It contains four major components with long unsaturated side chains of fifteen carbons: anacardic acid, cardanol, 2-methylcardol, and cardol, as shown in Figure $1^{[23,24]}$.

The final composition of CNSL depends on the oil extraction method. Natural CNSL mainly contains anacardic acid and is obtained from cold extraction. In hot extraction, anacardic acid is converted to cardanol with $90 \mathrm{wt} \%$ yield, giving so-called technical CNSL. The use of these natural phenolic compounds for the synthesis of epoxy, alkyd, and phenolic resins, miscellaneous coating materials, adhesives, and modifying agents for resins and plastics have been reported ${ }^{[25,26]}$. In addition, the authors reported that CNSL can act as a secondary dopant and plasticizer in the synthesis of PAni blends ${ }^{[27,28]}$.

The influence of CNSL on the mechanical and electrical properties of PAni.DBSA and styrene butadiene styrene tri-block copolymer (SBS) blends was evaluated. Results showed that the CNSL improved the mechanical and electrical properties due to the formation of cocontinuous-type morphology on the blend and a secondary doping process on DBSA-doped polyaniline ${ }^{[26]}$. Other researchers evaluated the action of the CNSL derivative, known as cardanol, functionalized with butane sulfonic acid, on the PAni synthesis. The functionalized cardanol acted as a dopant in the synthesis and promoted the formation of different nanostructures with electrical conductivities in the range of $10^{-1} \mathrm{~S} . \mathrm{cm}^{-1[27]}$. In a previous work, cardanol was used as a primary dopant for PAni, producing a polymer with electrical conductivity of $9.7 \times$

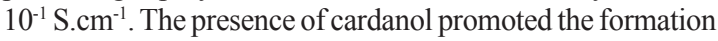
of PAni nanofibers mixed with particulates ${ }^{[29]}$.

Although studies indicate that CNSL can potentially improve the properties of PAni blends, the action of CNSL in the polymerization of aniline and its influence on the properties of the PAni thus obtained have not yet been investigated in detail. This study investigates the influence of technical CNSL on the morphology, solubility, and electrical and thermal properties of PAni synthesized by dispersion polymerization.

\section{Materials and Methods}

\subsection{Materials}

Aniline (analytical grade from Synth) was purified by distillation before polymerization. Hydrochloric acid $(\mathrm{HCl})$ (analytical grade from Neon), ammonium persulfate (APS)
(ACS reagent from Sigma-Aldrich), and cashew nut shell liquid (technical CNSL from Vernisul, Brazil) were used without further purification.

\subsection{Synthesis of doped polyaniline}

The doped PAni was synthesized in the absence and presence of CNSL by dispersion polymerization using $\mathrm{HCl}$ as a dopant and APS as an oxidant, according to the literature ${ }^{[19]}$. The $[\mathrm{HCl}] /[$ aniline] ratio was fixed at $3.3 \mathrm{HCl} 37 \mathrm{wt} \%$ (71.2 $\mathrm{mmol})$ and freshly distilled aniline $(21.48 \mathrm{mmol})$ were dispersed in $200 \mathrm{~mL}$ of distilled water. Then, APS (33.96 mmol) was added to the solution. The reaction occurred at $25{ }^{\circ} \mathrm{C}$ with continuous stirring for $60 \mathrm{~min}$. The dark green product was filtered and washed with ethanol to remove oligomers and dried for $48 \mathrm{~h}$ in a dessicator. By using the same methodology, a different amount of CNSL ( 25 and $40 \mathrm{wt} \%$ relative to the weight of aniline) was added to the mixture. The effects of experimental parameters such as the reaction time $(30,60$, and $120 \mathrm{~min})$ and temperature $\left(0{ }^{\circ} \mathrm{C}, 25^{\circ} \mathrm{C}\right.$, and $\left.55^{\circ} \mathrm{C}\right)$ were also investigated.

\subsection{Characterization}

Field-emission scanning electron microscopy (FESEM) was performed using an Inspect F50 microscope. The samples in powder form were metalized with gold. Transmission electron microscopy (TEM) was performed on a Tecnai GM2100F microscope with operating voltage of $80 \mathrm{kV}$. The electrical conductivity was determined by the four-point probe method (Keithley Instruments, model 236, and Multimeter HP34401) on pellet prepared by compacting the PAni powder. The absorption spectra of $\mathrm{KBr}$ pellets in the infrared region were obtained using a Perkin Elmer Spectrum One Fourier transform infrared spectrometer (FT-IR). UV-visible spectrometry (UV-vis) measurements were performed between 200 and $1100 \mathrm{~nm}$ using an HP Hewlett Packard 8453. Tests were conducted by preparing a PAni/ethanol solution for each sample. Thermogravimetric analysis (TGA) was performed using the TA Instruments Q60 under nitrogen atmosphere from $30^{\circ} \mathrm{C}$ to $800^{\circ} \mathrm{C}$ at a heating rate of $20^{\circ} \mathrm{C} / \mathrm{min}$. The crystallinity of the powdered form was measured using a Shimadzu $7000 \mathrm{X}$-ray diffractometer with $\mathrm{Cu} \mathrm{K} \alpha$ radiation $(\lambda=0.1540 \AA)$ at room temperature.

To evaluate the influence of CNSL on the solubility of PAni in water, the methodology described in the literature was adopted ${ }^{[15]}$. Doped PAni $(30 \mathrm{mg})$ was added to $30 \mathrm{~mL}$ of distilled water and ultrasonicated for $30 \mathrm{~min}$ at room temperature. Undissolved PAni was separated by filtration, dried at $100{ }^{\circ} \mathrm{C}$ in an oven for $1 \mathrm{~h}$, and finally weighed.

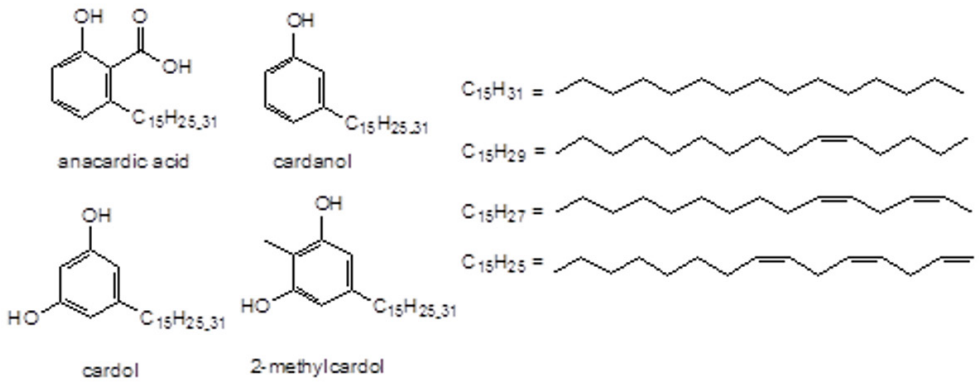

Figure 1. Major components of CNSL. 


\section{Results and Discussions}

\subsection{Influence of CNSL concentration}

The FT-IR spectrum of doped PAni (Figure 2) shows structural characteristics consistent with those of previous reports. The peak at $\sim 2915 \mathrm{~cm}^{-1}$ was attributed to the aliphatic $\mathrm{C}-\mathrm{H}$ stretching of CNSL ${ }^{[28]}$. The peaks at 1568 and $1487 \mathrm{~cm}^{-1}$ were attributed with the $\mathrm{C}=\mathrm{C}$ stretching modes of the quinoid and benzenoid units, respectively. The peak at $1294 \mathrm{~cm}^{-1}$ was attributed to the $\mathrm{C}-\mathrm{N}$ deformation of the benzenoid unit. The peak at $1118 \mathrm{~cm}^{-1}$ was attributed to the $\mathrm{C}-\mathrm{H}$ bending vibration of the $\mathrm{N}=\mathrm{Q}=\mathrm{N}$ segment ${ }^{[30,31]}$. The ratio between the intensity of the peaks at $1568 \mathrm{~cm}^{-1}$ (quinoid ring stretching-Q) and $1487 \mathrm{~cm}^{-1}$ (benzenoid ring stretching-B) was used to compare the doping efficiency between the samples ${ }^{[32]}$. According to the FT-IR spectra of the prepared samples, the ratio $\left[\mathrm{I}_{1487 \mathrm{~cm}-1}\right] /\left[\mathrm{I}_{1568 \mathrm{~cm}-1}\right]$ is close to 1 , confirming that the doped conductive PAni was in the emeraldine salt form.

Figure 3 shows the UV-Vis spectrum of the investigated samples. The entire spectra showed two bands at $340-370 \mathrm{~nm}$ and $400-440 \mathrm{~nm}$ and over $700 \mathrm{~nm}$. The first band was attributed to the $\pi-\pi^{*}$ electron transition of the benzenoid ring while the second and third bands correspond to the polaron $\pi^{*}$ transition and $\pi$-polaron transition ${ }^{[31,33]}$. Usually the first two bands are often combined into a flat or distorted single peak with a local maximum between the two peaks ${ }^{[34,35]}$. The intensity of the polaron bands absorption decreased with the introduction of the higher amount of CNSL. This phenomenon is related to the doping effectiveness of the polymer backbone ${ }^{[34]}$. The ratio between the polaron band absorbance $(790-850 \mathrm{~nm})$ and benzenoid $\pi-\pi *$ electron transition $(340-370 \mathrm{~nm})$ roughly estimate the level of PAni doping ${ }^{[34,36]}$. The values that were found were $0.91,0.90$ and 0.76 for $0 \%$ CNSL, 25\% CNSL and $40 \%$ CNSL, respectively. The sample with $40 \%$ CNSL shows the lowest level of doping and therefore is expected to have lower electrical conductivity.

The influence of CNSL on the morphology of PAni can be evaluated from FESEM micrographs. Figure 4 shows that PAni produced without CNSL showed a morphology predominantly formed by agglomerates, whereas an increase in the CNSL concentration favored the formation of nanofibers. The agglomerated morphology of synthesized PAni without

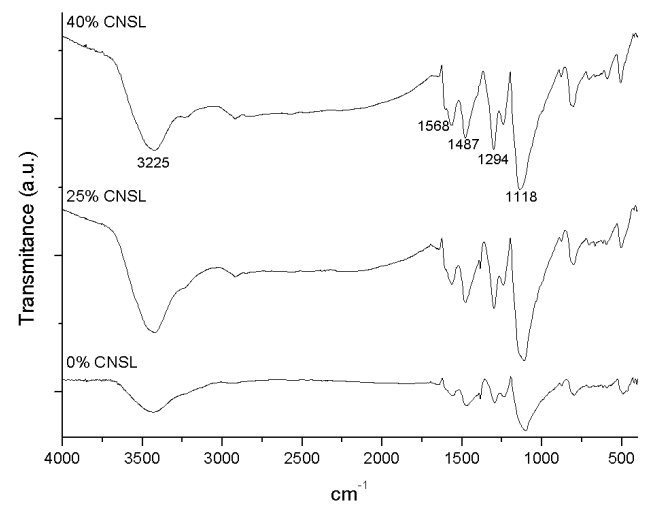

Figure 2. FT-IR spectrum of PAni nanofibers synthesized without and with CNSL. the addition of CNSL can be explained by the formation of unstable micelles of the dopant-aniline complex in water. The addition of CNSL can lead to an acid-base interaction between the CNSL and aniline salts in an aqueous medium reaction to form stable cylindrical micelles ${ }^{[27]}$. Therefore, CNSL, acts as a soft template and promotes the formation of PAni nanofibers with controlled dimensions through a self-assembly process ${ }^{[22]}$.

Figure 5 shows a TEM image of the PAni synthetized with 25\% CNSL. TEM image of PAni synthetized with 25\% CNSL shows dispersed and interconnected fibers with an average diameter of $(66 \pm 12) \mathrm{nm}$ and average length of $(1053 \pm 128) \mathrm{nm}$, resulting in an aspect ratio of $(18 \pm 6) \mathrm{nm}$.

Table 1 shows electrical conductivity values of PAni synthetized with and without CNSL. The conductivity values are of the same magnitude of those found in literature ${ }^{[1,21,22]}$. With the addition of CNSL, the electrical properties of PAni degrade yet not in a detrimental manner. This result is no surprise and can be explained by the chemical structure of CNSL. Specifically the CNSL has a long aliphatic side chain attached to an aromatic ring. Compounds with long side chains can act as plasticizers, increasing the spacing between polymer chains, thus decreasing in crystallinity and increasing solubility. An increase in the spacing between the polymer chains reduces the mobility of charge carriers ${ }^{[12]}$. In addition the insertion of CNSL also affects the doping efficiency of polyaniline as shown in Figure 3. These associated effects result in a decrease in electrical conductivity mainly for the sample containing $40 \%$ of CNSL.

The XRD scan in Figure 6 shows that the PAni prepared without CNSL exhibits four characteristic diffraction peaks

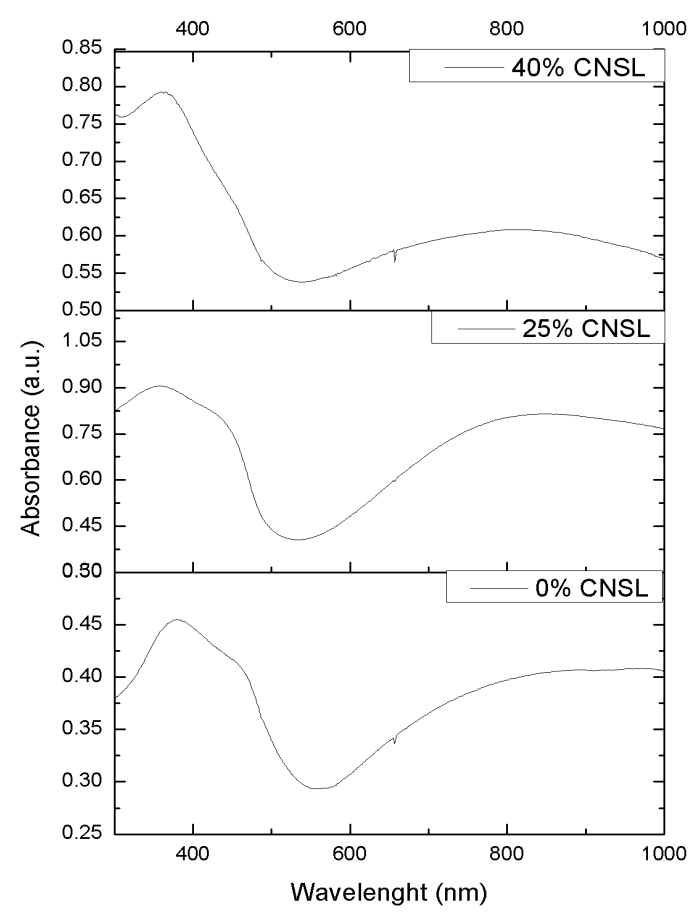

Figure 3. UV-vis absorption spectrum of PAni with and without CNSL. (a) $0 \%$ CNSL; (b) $25 \%$ CNSL, and (c) $40 \%$ CNSL. 


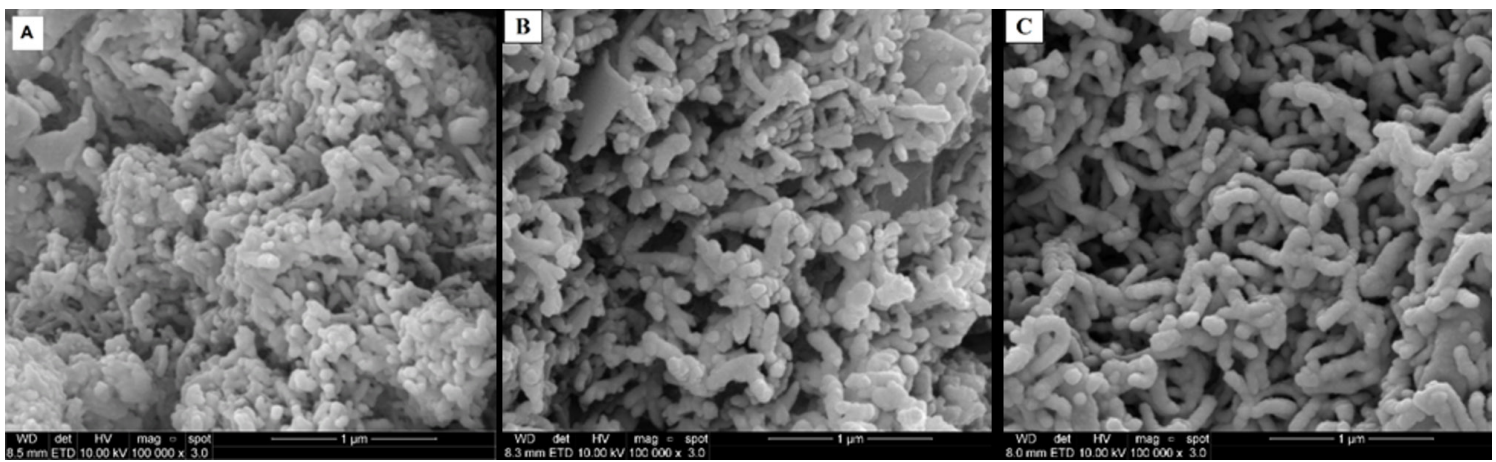

Figure 4. FESEM micrographs of synthetized PAni with (A) $0 \%$ CNSL, (B) $25 \%$ CNSL, and (C) $40 \%$ CNSL.

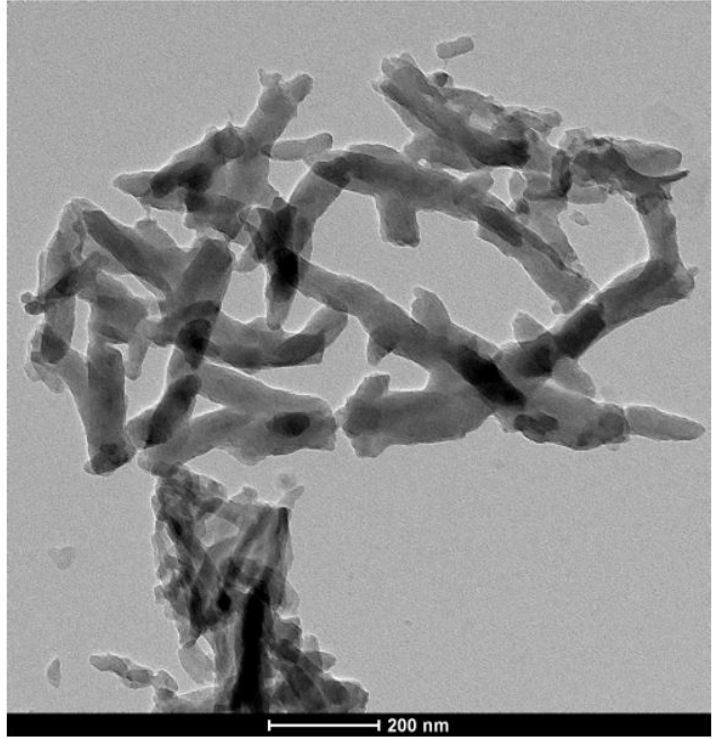

Figure 5. TEM micrograph of synthesized PAni with $25 \%$ CNSL.

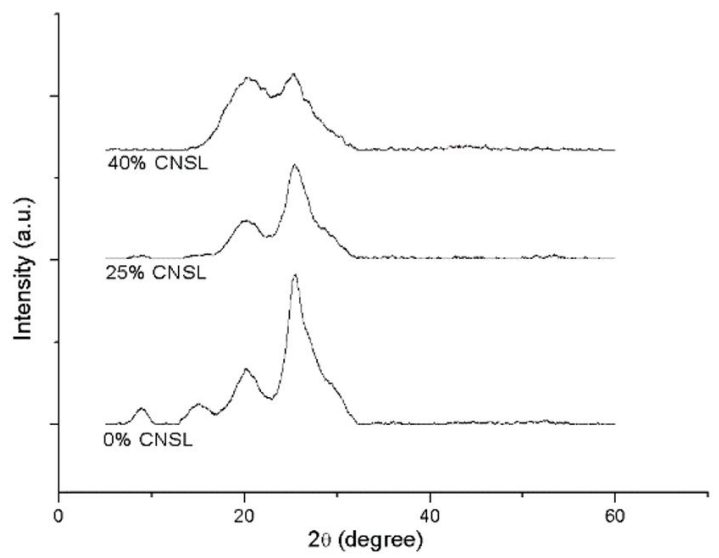

Figure 6. X-ray diffraction patterns of PAni nanofibers synthesized without and with CNSL.

with distinct intensities located at $2 \theta \cong 6^{\circ}, 15^{\circ}, 19^{\circ}$ and $25^{\circ}$ as described in literature ${ }^{[37-40]}$. The peaks located at $2 \theta \cong 6^{\circ}$ and $15^{\circ}$ disappear with the addition of CNSL, indicating a lower organization of the polymeric chain ${ }^{[40]}$. The ratio between the intensities of the peaks at $25^{\circ}$ and $19^{\circ},\left[\mathrm{I}_{25^{\circ}}\right]:\left[\mathrm{I}_{19^{\circ}}\right]$,
Table 1. Solubility, electrical, and thermal (as obtained by TGA) properties and XRD data of PAni synthetized without and with CNSL.

\begin{tabular}{cccccc}
\hline $\begin{array}{c}\text { CNSL } \\
(\%)\end{array}$ & $\begin{array}{c}\text { Electrical } \\
\text { Conductivity } \\
\left(\mathbf{S . c m}^{-1}\right)\end{array}$ & $\begin{array}{c}\mathbf{T}_{\text {onset }} \\
\left({ }^{\circ} \mathbf{C}\right)\end{array}$ & $\begin{array}{c}\mathbf{T}_{\max } \\
\left({ }^{\circ} \mathbf{C}\right)\end{array}$ & {$\left[\mathbf{I}_{25^{\circ}} \mathbf{0}:\left[\mathbf{I}_{19^{\circ}}\right]\right.$} & $\begin{array}{c}\text { Solubility } \\
\text { of PAni } \\
\text { in water } \\
(\%)\end{array}$ \\
\hline 0 & $2.6 \times 10^{0}$ & 327 & 408 & 6 & 8 \\
25 & $8.1 \times 10^{-1}$ & 350 & 443 & 2 & 94 \\
40 & $2.3 \times 10^{-3}$ & 352 & 447 & 1 & 97 \\
\hline
\end{tabular}

indicates the crystallinity of PAni; the higher the value of this ratio the better the ordering in the crystal structure ${ }^{[22]}$. Table 1 shows the ratios $\left[\mathrm{I}_{25^{\circ}}\right]:\left[\mathrm{I}_{19^{\circ}}\right]$ of the prepared samples. Higher crystallinity was observed in polymers prepared without the addition of CNSL. This is because CNSL can plasticize PAni, and thus, PAni nanofibers containing the highest amount of CNSL show lower crystallinity. These results are consistent with the electrical conductivity results. As discussed, lower crystallinity results in lower electrical conductivity $^{[41]}$.

The main disadvantages of conductive PAni in the emeraldine salt form are insolubility in water and conventional organic solvents and incompatibility with many conventional polymers owing to high aromaticity ${ }^{[32]}$. The solubility of the prepared PAni samples was evaluated, and the results are shown in Table 1 and Figure 7. Table 1 indicates that the presence of CNSL increased the solubility of PAni in water. The size of the CNSL side chains promotes interchain separation, facilitating the penetration of solvents molecules in clusters of polymer chains ${ }^{[12]}$.

Figure 8 shows TGA curves of the PAni nanofibers synthesized with different CNSL concentrations. PAni shows four stages of weight loss, independent of the experimental conditions. The initial weight loss between $50{ }^{\circ} \mathrm{C}$ and $100{ }^{\circ} \mathrm{C}$ can be attributed to the evaporation of water molecules. The second stage between $100{ }^{\circ} \mathrm{C}$ and $250{ }^{\circ} \mathrm{C}$ is due to free $\mathrm{HCl}$ (dopant unbound) and removal of low-molecular-weight oligomers ${ }^{[30,42]}$. The third stage between $250{ }^{\circ} \mathrm{C}$ and $300^{\circ} \mathrm{C}$ is attributed to loss of the primary dopant $\mathrm{HCl}^{[30,42-44]}$. The final stage between $300^{\circ} \mathrm{C}$ and $700^{\circ} \mathrm{C}$ is attributable to the degradation of the polymer ${ }^{[30,44-46]}$. PAni is completely oxidized at $\sim 700^{\circ} \mathrm{C}^{[30,46]}$. Table 1 shows that increasing the amount of CNSL in PAni nanofibers leads to an increase in the initial degradation temperature $\left(\mathrm{T}_{\text {onset }}\right)$ and maximum mass loss rate temperature $\left(\mathrm{T}_{\max }\right)$ relative to 
those of pure PAni. $\mathrm{T}_{\text {onset }}$ and $\mathrm{T}_{\max }$ increase by $\sim 25{ }^{\circ} \mathrm{C}$ and $\sim 40{ }^{\circ} \mathrm{C}$, respectively, when using $25 \% \mathrm{CNSL}$ and $40 \% \mathrm{CNSL}$. These results indicate that CNSL significantly improved the thermal stability of PAni nanofibers.

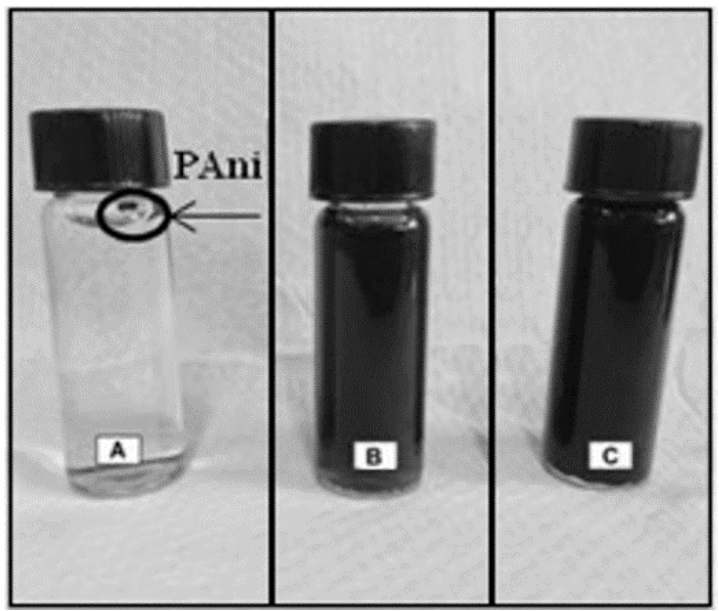

Figure 7. Solubility of different samples of PAni in water: (A) $0 \%$ CNSL, (B) $25 \%$ CNSL, and (C) $40 \%$ CNSL.

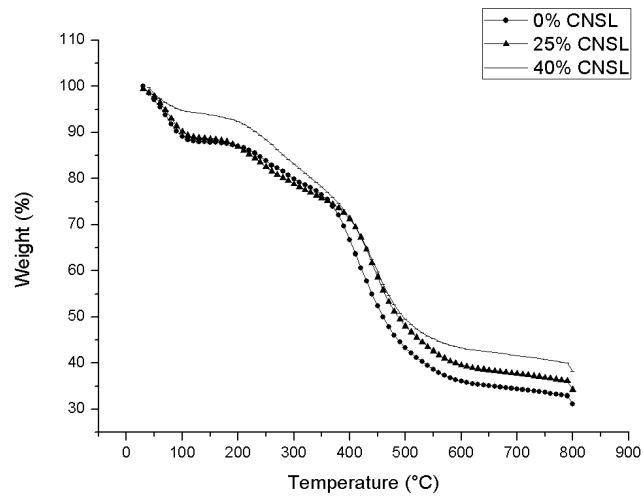

Figure 8. TGA plots of PAni samples.

\subsection{Influence of reaction time and temperature}

The morphology and electrical properties of PAni are strongly influenced by the experimental conditions. Therefore, the influence of temperature and reaction time on the properties of PAni nanofibers was evaluated ${ }^{[47,48]}$. As described above, adding 25\% CNSL improved the nanofiber morphology and thermal properties, and therefore, this experimental condition was fixed so as to evaluate the other parameters. Table 2 shows the evaluated parameters and results. To evaluate the influence of reaction time on the morphology and electrical properties of PAni nanofibers, small and large reaction time of 30 and $120 \mathrm{~min}$, respectively, were evaluated. In all cases, FTIR and UV-vis spectra (not presented here) confirmed the generation of PAni in its conductive emeraldine salt form.

Figure 9(a) and Table 2 indicate that PAni synthesized with a shorter reaction time of 30 min showed good-quality fibers but with low electrical conductivity of $8.7 \times 10^{-4} \mathrm{~S}^{\mathrm{cm}} \mathrm{cm}^{-1}$. This is because the reaction time was not sufficient for fully doping the polymer, leading to a low degree of protonation. On the other hand, Figure 9(b) and Table 2 indicate that a longer reaction time of $120 \mathrm{~min}$ favored the formation of agglomerates and reduced electrical conductivity. This is because of secondary growth during polymerization. The longer the reaction time, the higher is the probability of the PAni polymeric chain becoming a support for the growth of irregular structures ${ }^{[49]}$. As shown in Figure 4(b) and Table 2, a reaction time of $60 \mathrm{~min}$ is sufficient to

Table 2. Electrical conductivity under different experimental conditions.

\begin{tabular}{cccc}
\hline $\begin{array}{c}\text { Reaction time } \\
(\mathbf{m i n})\end{array}$ & $\begin{array}{c}\text { Temperature } \\
\left({ }^{\circ} \mathbf{C}\right)\end{array}$ & $\begin{array}{c}\text { Average } \\
\text { diameter }(\mathbf{n m})\end{array}$ & $\begin{array}{c}\text { Electrical } \\
\text { conductivity } \\
\left(\mathbf{S . c m}^{-1}\right)\end{array}$ \\
\hline 30 & 25 & $64 \pm 11$ & $8.7 \times 10^{-4}$ \\
60 & 25 & $66 \pm 12$ & $8.1 \times 10^{-2}$ \\
120 & 25 & $74 \pm 15$ & $1.3 \times 10^{-4}$ \\
60 & 0 & $85 \pm 14$ & $1.1 \times 10^{-4}$ \\
60 & 55 & $68 \pm 13$ & $1.0 \times 10^{-3}$ \\
\hline
\end{tabular}

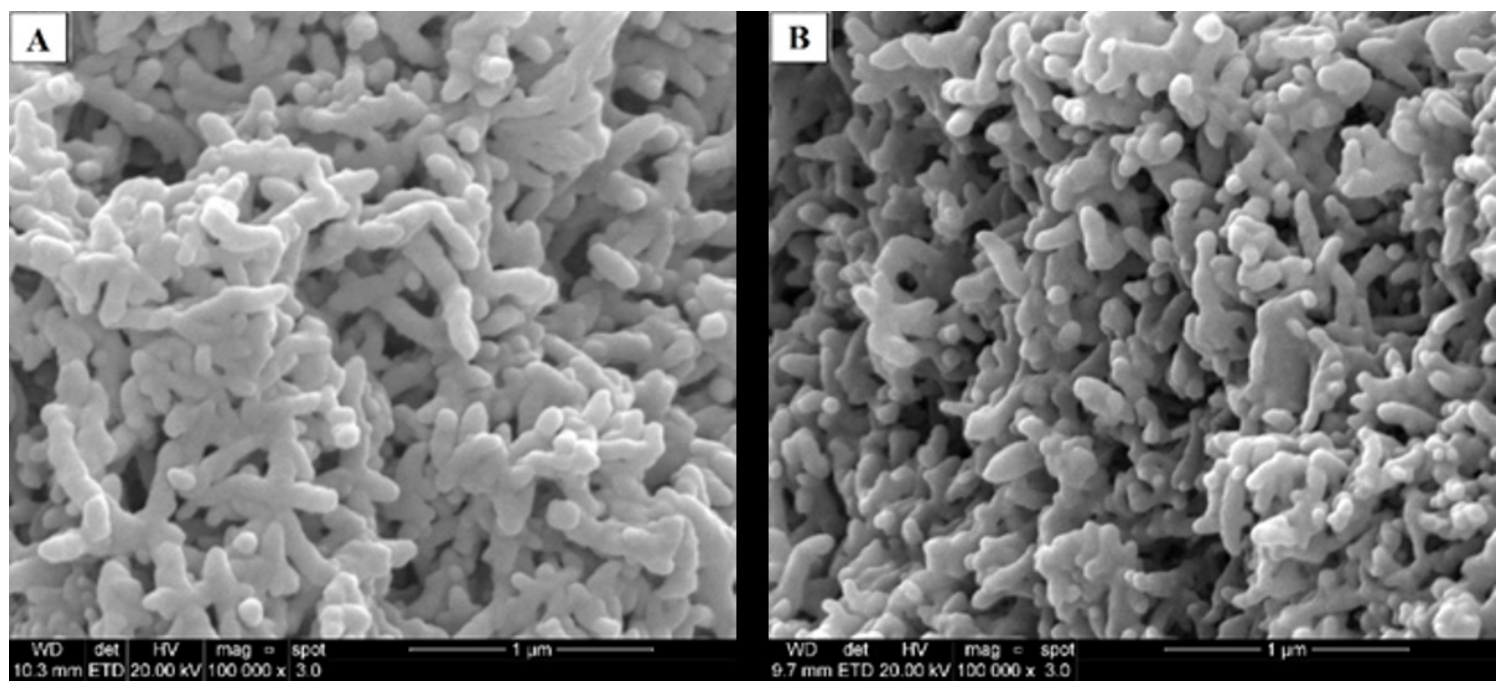

Figure 9. FESEM micrographs with reaction time of (A) $30 \mathrm{~min}$ and (B) $120 \mathrm{~min}$. 

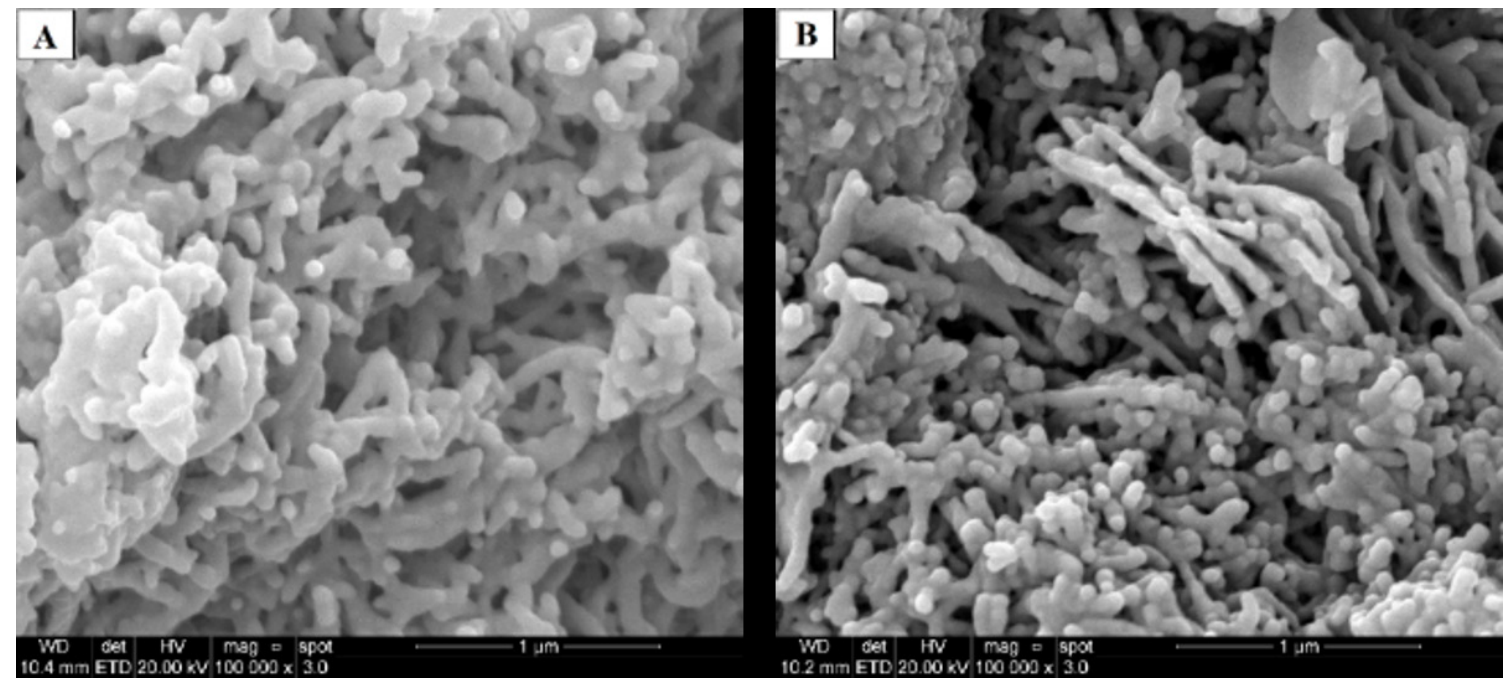

Figure 10. FESEM micrographs of PAni synthesized at temperature of (A) $0^{\circ} \mathrm{C}$ and (B) $55^{\circ} \mathrm{C}$.

produce conductive polymer nanofibers with well-defined morphology.

Polymerization was performed at three different temperatures: $0{ }^{\circ} \mathrm{C}, 25^{\circ} \mathrm{C}$, and $55^{\circ} \mathrm{C}$. FESEM images showed the presence of aggregates of PAni nanofibers for reactions at temperatures of $0^{\circ} \mathrm{C}$ and $55^{\circ} \mathrm{C}$ (Figure 10 (a) and (b), respectively), whereas the reaction at $25^{\circ} \mathrm{C}$ generated high-quality nanofibers (Figure 4(b)). The electrical conductivity was also sensitive to temperature. The sample prepared at $25^{\circ} \mathrm{C}$ showed electrical conductivity that was 100 and 10 times higher than that of samples synthesized at $0^{\circ} \mathrm{C}$ and $55^{\circ} \mathrm{C}$, respectively, as shown in Table 2 .

\section{Conclusions}

Adding CNSL during the synthesis of PAni promoted the formation of nanofibers and increased the thermal stability. Moreover, CNSL appears to be an effective surfactant that promotes and stabilizes the dispersion of PAni nanofibers in water, allowing ecofriendly synthesis. The presence of CNSL promotes the plasticization of PAni nanofibers, thus degrading the electrical properties. Nevertheless, PAni nanofibers remain semiconductive even at high CNSL concentrations (i.e., 40\%). Nanofibers with the best conductivity and most well-defined morphology were obtained when the reaction occurred at $25{ }^{\circ} \mathrm{C}$ for $60 \mathrm{~min}$. Reaction times of 30 or $120 \mathrm{~min}$ and temperatures of $0{ }^{\circ} \mathrm{C}$ or $55^{\circ} \mathrm{C}$ resulted in the formation of agglomerates and reduced the electrical conductivity. These results clearly indicate that the lateral aliphatic chain allows CNSL to act as a soft template, thus improving the PAni morphology and increasing the surface area of the polymer. Thus, CNSL, a biodegradable and renewable resource, can potentially expand the technological applications of PAni nanofibers.

\section{Acknowledgements}

This work was support by Coordenação de Aperfeiçoamento de Pessoal de Nível Superior (CAPES) and Conselho Nacional de Desenvolvimento Científico e
Tecnológico (CNPq). The authors acknowledge VERNISUL by supply of the technical CNSL.

\section{References}

1. Simões, F. R., Bulhões, L. O. S., \& Pereira, E. C. (2009). Synthesis and characterization of conducting composites of polyaniline and carbon black with high thermal stability. Polimeros: Ciência e Tecnologia, 19(1), 54-57. http://dx.doi. org/10.1590/S0104-14282009000100013

2. Hacıvelioğlu, F., Kılıç, N., Çelebi, E. B., \& Yeşilot, S. (2016). In situ preparation and properties of sulfonic and phosphonic acid substituted polyphosphazene/polyaniline composites. Polymer, 96, 112-120. http://dx.doi.org/10.1016/j.polymer.2016.05.008.

3. Wang, C., Wang, L., Jin, J., Liu, J., Li, Y., Wu, M., Chen, L., Wang, B., Yang, X., \& Su, B. (2016). Probing effective photocorrosion inhibition and highly improved photocatalytic hydrogen production on monodisperse PANI@CdS core-shell nanospheres. Applied Catalysis B: Environmental, 188, 351359. http://dx.doi.org/10.1016/j.apcatb.2016.02.017.

4. Gu, Z., Zhang, Q., \& Shen, Q. (2015). Synthesis and comparison of polyaniline nanofibers templated by $\alpha-, \beta$ - and $\gamma$-cyclodextrin. Journal of Polymer Research, 2015(22), 7. http://dx.doi. org/10.1007/s10965-014-0647-5.

5. Summers, G. J., Waware, U. S., Maduwa, M. R., \& Summers, C. A. (2015). Conducting polyaniline nanorods doped with aromatic carboxyl chain end functionalized polystyrene. Synthetic Metals, 209, 251-261. http://dx.doi.org/10.1016/j. synthmet.2015.07.026.

6. Chutia, P., \& Kumar, A. (2015). Electrical transport and morphological studies of polyaniline nanostructures. Polymer Engineering and Science, 55(5), 995-1002. http://dx.doi. org/10.1002/pen.23967.

7. Chen, X., Li, H., Wu, H., Wu, Y., Shang, Y., Pan, J., \& Xiong, X. (2016). Fabrication of TiO2@PANI nanobelts with the enhanced absorption and photocatalytic performance under visible light. Materials Letters, 172, 52-55. http://dx.doi. org/10.1016/j.matlet.2016.02.134

8. Han, J., Song, G., \& Guo, R. (2007). Nanostructure-Based Leaflike Polyaniline in the Presence of an Amphiphilic Triblock Copolymer. Advanced Materials, 19(19), 2993-2999. http:// dx.doi.org/10.1002/adma.200602635. 
9. Yang, N., Zhai, J., Wan, M., Wang, D., \& Jiang, L. (2010). Layered nanostructures of polyaniline with graphene oxide as the dopant and template. Synthetic Metals, 160(15-16), 1617-1622. http://dx.doi.org/10.1016/j.synthmet.2010.05.029.

10. Dallas, P., \& Georgakilas, V. (2015). Interfacial polymerization of conductive polymers: generation of polymeric nanostructures in a 2-D space. Advances in Colloid and Interface Science, 224, 46-61. PMid:26272721. http://dx.doi.org/10.1016/j. cis.2015.07.008

11. Yin, H., \& Yang, J. (2011). Synthesis of high-performance onedimensional polyaniline nanostructures using dodecylbenzene sulfonic acid as soft template. Materials Letters, 65(5), 850-853. http://dx.doi.org/10.1016/j.matlet.2010.12.031.

12. Sinha, S., Bhadra, S., \& Khastgir, D. (2009). Effect of dopant type on the properties of polyaniline. Journal of Applied Polymer Science, 112(5), 3135-3140. http://dx.doi.org/10.1002/ app.29708.

13. Ćirić-Marjanović, G. (2013). Recent advances in polyaniline research: polymerization mechanisms, structural aspects, properties and applications. Synthetic Metals, 177, 1-47. http:// dx.doi.org/10.1016/j.synthmet.2013.06.004.

14. Pahovnik, D., Žagar, E., Kogej, K., Vohlídal, J., \& Žigon, M. (2013). Polyaniline nanostructures prepared in acidic aqueous solutions of ionic liquids acting as soft templates. European Polymer Journal, 49(6), 1381-1390. http://dx.doi.org/10.1016/j. eurpolymj.2013.02.019.

15. Wang, Y., Chen, K., Li, T., Li, H., Zeng, R., Zhang, R., Gu, Y., Ding, J., \& Liu, H. (2014). Soluble polyaniline nanofibers prepared via surfactant-free emulsion polymerization. Synthetic Metals, 198, 293-299. http://dx.doi.org/10.1016/j. synthmet.2014.10.038.

16. Zhang, Z., Wei, Z., \& Wan, M. (2002). Nanostructures of polyaniline doped with inorganic acids. Macromolecules, 35(15), 5937-5942. http://dx.doi.org/10.1021/ma020199v.

17. Zhang, Z., Wei, Z., Zhang, L., \& Wan, M. (2005). Polyaniline nanotubes and their dendrites doped with different naphthalene sulfonic acids. Acta Materialia, 53(5), 1373-1379. http://dx.doi org/10.1016/j.actamat.2004.11.030.

18. Anilkumar, P., \& Jayakannan, M. (2007). Fluorescent tagged probing agent and structure-directing amphiphilic molecular design for polyaniline nanomaterials via self-assembly process. The Journal of Physical Chemistry C, 111(9), 3591-3600. http:// dx.doi.org/10.1021/jp066428n

19. Jang, J., Bae, J., \& Lee, K. (2005). Synthesis and characterization of polyaniline nanorods as curing agent and nanofiller for epoxy matrix composite. Polymer, 46(11), 3677-3684. http:// dx.doi.org/10.1016/j.polymer.2005.03.030.

20. Ayad, M. M., Salahuddin, N. A., Minisy, I. M., \& Amer, W. A. (2014). Chitosan/polyaniline nanofibers coating on the quartz crystal microbalance electrode for gas sensing. Sensors and Actuators. B, Chemical, 202, 144-153. http://dx.doi. org/10.1016/j.snb.2014.05.046.

21. Qiu, H., Qi, S., Wang, D., Wang, J., \& Wu, X. (2010). Synthesis of polyaniline nanostructures via soft template of sucrose octaacetate. Synthetic Metals, 160(11-12), 1179-1183. http:// dx.doi.org/10.1016/j.synthmet.2010.03.005.

22. Grover, S., Goel, S., Marichi, R. B., Sahu, V., Singh, G., \& Sharma, R. K. (2016). Polyaniline all solid-state pseudocapacitor: role of morphological variations in performance evolution. Electrochimica Acta, 196, 131-139. http://dx.doi.org/10.1016/j. electacta.2016.02.157.

23. Lochab, B., Shukla, S., \& Varma, I. K. (2014). Naturally occurring phenolic sources: monomers and polymers. RSC Advances, 4(42), 21712-21752. http://dx.doi.org/10.1039/ C4RA00181H.
24. Voirin, C., Caillol, S., Sadavarte, N. V., Tawade, B. V., Boutevin, B., \& Wadgaonkar, P. P. (2014). Functionalization of cardanol: towards biobased polymers and additives. Polymer Chemistry, 5(9), 3142-3162. http://dx.doi.org/10.1039/C3PY01194A.

25. Balachandran, V. S., Jadhav, S. R., Vemula, P. K., \& John, G. (2013). Recent advances in cardanol chemistry in a nutshell: from a nut to nanomaterials. Chemical Society Reviews, 42(2), 427-438. PMid:23114456. http://dx.doi.org/10.1039/ C2CS35344J.

26. Souza, F. G. Jr, Pinto, J. C., Oliveira, G. E., \& Soares, B G. (2007). Evaluation of electrical properties of SBS/Pani blends plasticized with DOP and CNSL using an empirical statistical model. Polymer Testing, 26(6), 720-728. http:// dx.doi.org/10.1016/j.polymertesting.2007.03.004

27. Anilkumar, P., \& Jayakannan, M. (2009). Self-assembled cylindrical and vesicular molecular templates for polyaniline nanofibers and nanotapes. The Journal of Physical Chemistry $B, 113(34), 11614-11624$. PMid:19642663. http://dx.doi. org/10.1021/jp9043418.

28. Srivastava, R., \& Srivastava, D. (2015). Mechanical, chemical, and curing characteristics of cardanol-furfural-based novolac resin for application in green coatings. Journal of Coatings Technology and Research, 12(2), 303-311. http://dx.doi org/10.1007/s11998-014-9630-7.

29. Gonçalves, R. V., Zanini, M. L., Malmonge, J. A., Bonnaud, L., \& Basso, N. R. S. (2016). Pristine cardanol as biobased dopant for polyaniline. Materials Letters, 185, 327-330. http:// dx.doi.org/10.1016/j.matlet.2016.07.138.

30. Basso, N. R. S., Oliveira, F., Graebin, A. P., Moura, C. S., Fim, F. C., Galland, G. B., Bonnaud, L., Murariu, O., \& Dubois, P. (2014). Polyaniline nanofibers for In situ MAO-catalyzed polymerization of ethylene. Journal of Applied Polymer Science, 131, 41197.

31. Li, Y., Li, G., Peng, H., Qin, Y., \& Chen, K. (2013). Facile synthesis of high-quality ultralong poly(aniline-co-pphenylenediamine) nanofibers. Synthetic Metals, 164, $42-46$. http://dx.doi.org/10.1016/j.synthmet.2012.12.024.

32. Silva, M. J., Sanches, A. O., Malmonge, L. F., Medeiros, E. S., Rosa, M. F., McMahan, C. M., \& Malmonge, J. A. (2012). Conductive nanocomposites based on cellulose nanofibrils coated with polyaniline-dbsa via in situ polymerization. Macromolecular Symposia, 319(1), 196-202. http://dx.doi. org/10.1002/masy.201100156.

33. Xia, H., \& Wang, Q. (2001). Synthesis and characterization of conductive polyaniline nanoparticles through ultrasonic assisted inverse microemulsion polymerization. Journal of Nanoparticle Research, 3(5/6), 401-411. http://dx.doi. org/10.1023/A:1012564814745.

34. Han, M. G., Cho, S. K., Oh, S. G., \& Im, S. S. (2002). Preparation and characterization of polyaniline nanoparticles synthesized from DBSA micellar solution. Synthetic Metals, 126(1), 53-60. http://dx.doi.org/10.1016/S0379-6779(01)00494-5.

35. Stejskal, J., Kratochvíl, P., \& Radhakrishnan, N. (1993). Polyaniline dispersions 2. UV-Vis absorption spectra. Synthetic Metals, 61(3), 225-231. http://dx.doi.org/10.1016/03796779(93)91266-5.

36. Han, Y. G., Kusunose, T., \& Sekino, T. (2009). One-step reverse micelle polymerization of organic dispersible polyaniline nanoparticles. Synthetic Metals, 159(1-2), 123-131. http:// dx.doi.org/10.1016/j.synthmet.2008.08.011.

37. Neelgund, G. M., Bliznyuk, V. N., \& Oki, A. (2016). Photocatalytic activity and NIR laser response of polyaniline conjugated graphene nanocomposite prepared by a novel acid-less method. Applied Catalysis B: Environmental, 187, 357-366. PMid:26917864. http://dx.doi.org/10.1016/j.apcatb.2016.01.009. 
38. Yan, Q., Wang, M., Wu, Y., \& Shen, Q. (2016). Tea polyphenol as environmentally friendly dopant and thermal stabilizer for polyaniline. Materials Letters, 170, 202-204. http://dx.doi. org/10.1016/j.matlet.2016.02.041.

39. Oliveira, L. R., Manzato, L., Mascarenhas, Y. P., \& Sanches, E. A. (2017). The influence of heat treatment on the semicrystalline structure of polyaniline Emeraldine-salt form. Journal of Molecular Structure, 1128, 707-717. http://dx.doi. org/10.1016/j.molstruc.2016.09.044.

40. Sanches, E. A., Soares, J. C., Mafud, A. C., Fernandes, E. G. R., Leite, F. L., \& Mascarenhas, Y. P. (2013). Structural characterization of Chloride Salt of conducting polyaniline obtained by XRD, SAXD, SAXS and SEM. Journal of Molecular Structure, 1036, 121-126. http://dx.doi.org/10.1016/j. molstruc.2012.09.084.

41. Bhadra, S., \& Khastgir, D. (2009). Glass-rubber transition temperature of polyaniline: experimental and molecular dynamic simulation. Synthetic Metals, 159(12), 1141-1146. http://dx.doi.org/10.1016/j.synthmet.2009.01.052.

42. Zhao, W., Ma, L., \& Lu, K. (2007). Facile synthesis of polyaniline nanofibers in the presence of polyethylene glycol. Journal of Polymer Research, 14(1), 1-4. http://dx.doi.org/10.1007/ s10965-006-9069-3.

43. Sen, T., Mishra, S., \& Shimpi, N. G. (2017). A b-cyclodextrin based binary dopant for polyaniline: structural, thermal, electrical, and sensing performance. Materials Science and Engineering B, 220, 13-21. http://dx.doi.org/10.1016/j.mseb.2017.03.003.

44. Yan, J., Huang, Y., Wei, C., Zhang, N., \& Liu, P. (2017). Covalently bonded polyaniline/graphene composites as highperformance electromagnetic (EM) wave absorption materials. Composites.
Part A, Applied Science and Manufacturing, 99, 121-128. http:// dx.doi.org/10.1016/j.compositesa.2017.04.016.

45. Bhadra, S., \& Khastgir, D. (2008). Extrinsic and intrinsic structural change during heat treatment of polyaniline. Polymer Degradation \& Stability, 93(6), 1094-1099. http://dx.doi. org/10.1016/j.polymdegradstab.2008.03.013.

46. Nobrega, M. M., Izumi, C. M. S., \& Temperini, M. L. A. (2015). Probing molecular ordering in the HCl-doped polyaniline with bulk and nanofiber morphology by their thermal behavior. Polymer Degradation \& Stability, 113, 66-71. http://dx.doi. org/10.1016/j.polymdegradstab.2015.01.015.

47. Ležaić, A. J., Bajuk-Bogdanović, D., Radoičić, M., Mirsky, V. M., \& Ćirić-Marjanović, G. (2016). Influence of synthetic conditions on the structure and electrical properties of nanofibrous polyanilines and their nanofibrous carbonized forms. Synthetic Metals, 214, 35-44. http://dx.doi.org/10.1016/j. synthmet.2016.01.015.

48. Krukiewicz, K., \& Katunin, A. (2016). The effect of reaction medium on the conductivity and morphology of polyaniline doped with camphorsulfonic acid. Synthetic Metals, 214, 4549. http://dx.doi.org/10.1016/j.synthmet.2016.01.017.

49. Huang, J., \& Kaner, R. B. (2004). A general chemical route to polyaniline nanofibers. Journal of the American Chemical Society, 126(3), 851-855. PMid:14733560. http://dx.doi. org/10.1021/ja0371754.

Received: Mar: 13, 2017 Revised: May 11, 2017 Accepted: May 12, 2017 